\title{
Design of Wavelet-Encoded Symbol Constellations for Cyclostationary Spectrum Sensing
}

\author{
Pedro Thiago Valério de Souza ${ }^{D}{ }^{1}{ }^{1}$ Vinícius Samuel Valério de Souza, \\ and Luiz Felipe de Queiroz Silveira $\mathbb{D}^{2}$ \\ ${ }^{1}$ Departamento de Engenharias e Tecnologia, UFERSA, Pau Dos Ferros/RN, Brazil \\ ${ }^{2}$ Departamento de Engenharia de Computação e Automação, UFRN, Natal/RN, Brazil \\ Correspondence should be addressed to Pedro Thiago Valério de Souza; pedro.souza@ufersa.edu.br
}

Received 4 August 2019; Revised 20 November 2019; Accepted 23 December 2019; Published 21 January 2020

Academic Editor: Miguel López-Benítez

Copyright (c) 2020 Pedro Thiago Valério de Souza et al. This is an open access article distributed under the Creative Commons Attribution License, which permits unrestricted use, distribution, and reproduction in any medium, provided the original work is properly cited.

\begin{abstract}
In mobile communication systems, the signals propagate through multipath over time-varying channels, which are subject to distortion caused by fading and Doppler shifts. In order to minimize such distortions, coding techniques and transmission diversity can be employed, e.g., wavelet coding. In this work, the wavelet coding is investigated in scenarios of cognitive radio systems with dynamic spectrum access. Cognitive radio systems with dynamic spectrum access should be able to sense unoccupied frequency bands for opportunistic transmissions, as well as detect the presence of primary users when they occupy their licensed spectrum. Therefore, an essential element for the accurate operation of cognitive radio systems encoded by wavelet coding is the ability to sense the signals encoded by this technique. It is effectively demonstrated that the possibility of sensing such signals is associated with a suitable design of the signal constellation used in the modulation of the coded symbols. The constellation design of these is performed via genetic algorithms using a multiobjective optimization approach. The developed system is evaluated according to the robustness to time-varying flat fading through a bit error probability (BER) versus $E_{b} / N_{0}$ analysis. The spectral sensing ability is also addressed employing the cyclostationary analysis. The results denote the feasibility of using wavelet coding in radio scenarios with dynamic spectrum access, with good performance in terms of BER and signal detection rates.
\end{abstract}

\section{Introduction}

In wireless communication channels, the information signals propagate between the transmitting antenna and the receiving antenna through channels characterized by timevarying fading, which deteriorates the transmitted signals. One possible way to overcome such undesirable effect is to provide transmission diversity [1].

Transmission diversity techniques aim at transmitting signal replicas over uncorrelated channels. Thus, the greater the number of replicas, the greater the system diversity, while improved performance results if compared with the case where a single signal is transmitted [2]. In general, transmission diversity techniques reduce the spectral efficiency of the system [3]. However, when such diversity is provided by appropriate techniques, prominent advantages can be achieved by maintaining the spectral efficiency of the system at appropriate values. Wavelet coding is a transmission technique that can be used for this purpose [4].

Wavelet coding was initially proposed by Tzannes and Tzannes [5], being characterized by using wavelet matrices for information coding [2]. Because of the orthogonality property of the rows in the wavelet matrices, the information can be retrieved at the receiver through a bank of correlators that match the rows used for coding [4].

Simplicity regarding the wavelet coding process led to the investigation of this transmission technique applied to different communication scenarios, such as flat fading channels [6], frequency selective channels [4], analysis of channel estimation errors $[7,8]$, and identification of modulation schemes designed by genetic algorithms [9], in 
OFDM systems on a 4.9 Gbps W-Band Radio-over-Fiber Link [10] and in wireless adaptive control systems [11].

In fact, the computational complexity of wavelet coding is linear with increasing number of coding matrix columns [12]. On the other hand, wavelet coding has already been successfully used in real systems with small and medium size matrices. In [10], a $2 \times 128$-size matrix was used to enable communications on an $80 \mathrm{GHz}$ carrier frequency real fiber radio system. In [11], a small $(2 \times 8)$ matrix was used to enable a microcontrolled wireless loop control system. However, the wavelet coding technique has not yet been investigated in scenarios where systems employ spectrum sensing and dynamic spectrum access.

Spectrum sensing is a task for which the radio system must detect free portions of the electromagnetic spectrum. One of the main advantages associated with spectrum sensing is the improved management of the electromagnetic spectrum. In particular, scientific studies have shown that the spectrum access problem is more significant than the physical scarce availability of this resource [13]. In this context, regulatory agencies have proposed a new spectrum allocation policy called dynamic spectrum access (DSA). In this case, the radio system must be capable of detecting free portions through spectral sensing techniques.

In general, three classical techniques are defined in the literature for spectral sensing: energy detection, cyclostationary analysis, and matched filters. In particular, cyclostationarity sensing stands out as the most efficient approach for detecting signals in environments with low signal-tonoise ratio (SNR), especially because it does require the prior knowledge of the signals to be sensed [14].

The possibility of detecting users through coding with wavelet matrices is of fundamental importance for the application of this coding technique to systems employing spectrum sensing. However, as it will be further demonstrated in this work, there are practical difficulties when applying cyclostationarity-based spectrum sensing techniques to wavelet-encoded signals. This is due to the fact that wavelet symbols are modulated and transmitted over the communication channel.

In this context, this work aims at adapting the wavelet coding to the DSA scenario using spectrum sensing by cyclostationarity analysis, without compromising the robustness provided by such transmission technique. In particular, wavelet coding becomes suitable to this application by adjusting the way the generated symbols are modulated by coding. The wavelet symbols are modulated from a phase-shift keying (PSK) constellation optimized via a genetic algorithm in order to meet the requirements of simple detection of encoded signals and robustness to fading effects, which is evaluated in terms of the bit error rate (BER).

The remainder of the paper is organized as follows. Section 2 introduces the fundamental concepts of wavelet coding. Section 3 presents the theory regarding cyclostationary spectrum sensing. Section 4 describes the design process of constellations that are suitable for spectrum sensing of wavelet coded symbols. Section 5 presents the simulation results, while Section 6 discusses the main conclusions of this work.

\section{Concepts of Wavelet Coding}

Let us consider matrix $\mathbf{A}=\left(a_{k}^{s}\right)$ described by equation (1), which has $m$ rows $(m \geq 2)$ and $m g$ columns, whose elements belong to the set $\{+1,-1\}$ :

$$
\mathbf{A}=\left[\begin{array}{cccc}
a_{0}^{0} & a_{1}^{0} & \cdots & a_{m g-1}^{0} \\
a_{0}^{1} & a_{0}^{1} & \cdots & a_{m g-1}^{1} \\
\vdots & \vdots & \ddots & \vdots \\
a_{0}^{m-1} & a_{1}^{m-1} & \cdots & a_{m g-1}^{m-1}
\end{array}\right],
$$

where $\mathbf{A}$ is defined as the wavelet coefficient matrix (WCM), which is an integer and plane with rank $m$ and gender $g$ if the conditions given in equations (2) and (3) are satisfied [2]

$$
\sum_{k=0}^{m g-1} a_{k}^{s}=m \sqrt{g} \delta(s, 0), \quad 0 \leq s \leq m-1,
$$

$$
\begin{aligned}
\sum_{k=0}^{m g-1} a_{\left[k+m r^{\prime}\right]}^{s}\left(a_{[k+m r]}^{s}\right)= & m g \delta\left(s^{\prime}, s\right) \delta\left(r^{\prime}, r\right), \\
& s^{\prime} \geq 0, s \leq m-1, r^{\prime} \geq 0, r \leq g-1,
\end{aligned}
$$

where $[k+m r]$ is the result of operation $k+m r$ modulus $m g$, $a^{*}$ is the complex conjugate of $a$, and $\delta(x, y)$ is the Kronecker delta function, which is defined by [2]

$$
\delta(x, y)= \begin{cases}0, & \text { if } x \neq y \\ 1, & \text { otherwise }\end{cases}
$$

The condition given by equation (3) denotes that the rows of a wavelet matrix with rank $m$ and gender $g$ have a norm $m^{1 / 2}$ and are mutually orthogonal to each other, even when shifted by a multiple of $m$. Equation (2) shows that the sum of the elements in the first row of the wavelet matrix is equal to the matrix rank, while the sum of the elements in each one of the remaining rows is zero [4].

2.1. Wavelet Encoding. Let us consider a discrete source that generates symbols $x_{i} \in\{1,-1\}$, which are statistically independent and have equal probability of occurrence. The wavelet coding can be defined mathematically by the matrix product as [5]

$$
\mathbf{Y}=\mathbf{C}_{\mathrm{WCM}} \mathbf{X}
$$

where $\mathbf{Y}$ is a vector with $N$ coded symbols referred to as wavelet symbols $y_{n}, \mathbf{X}$ is a vector composed of a sequence with $N$ information symbols $x_{i}$, and $\mathbf{C}_{\mathrm{WCM}}$ is a transformation matrix with $N$ rows, which is obtained from successive repetitions and shifting of the WCM in order to maintain the orthogonality as defined by equation (3). Equation (6) presents an example of $\mathbf{C}_{\mathrm{WCM}}$ obtained from a $2 \times 8$ WCM [4]: 


$$
\mathrm{C}_{\mathrm{WCM}}=\left[\begin{array}{ccccccc}
a_{0}^{0} & a_{1}^{0} & a_{2}^{0} & \cdots & a_{7}^{0} & & \\
a_{0}^{1} & a_{1}^{1} & a_{1}^{1} & \cdots & a_{7}^{1} & & \\
& & a_{0}^{0} & a_{1}^{0} & a_{2}^{0} & \cdots & a_{7}^{0} \\
& & a_{0}^{1} & a_{1}^{1} & a_{1}^{1} & \cdots & a_{7}^{1} \\
& & & & \vdots & \ddots & \vdots
\end{array}\right] .
$$

It is possible to demonstrate that the wavelet symbols $y_{p m+q}$ transmitted at time instant $n=p m+q$, where $p \in\{0, \ldots,(N-m) / m\}$ and $q \in\{0, \ldots, m-1\}$, can be defined by $[4]$

$$
y_{p m+q}=\sum_{j=0}^{m-1} \sum_{l=0}^{g-1} a_{l m+q}^{j} x_{(p-l) m+j},
$$

and belong to the set

$$
y_{n} \in\{-m g,-m g+2, \ldots, 0, \ldots, m g-2, m g\},
$$

which has a cardinality $m g+1$ and a probability mass function given by [2]

$$
P_{r}\left(y_{n}=2 k-m g\right)=\left(\begin{array}{c}
m g \\
k
\end{array}\right) 0.5^{m g}
$$

where the mean is zero and the variance is $m g$.

2.2. Wavelet Decoding. Decoding the wavelet symbols $y_{n}$ is based on the orthogonality property of the WMC rows, which can be defined from the matrix product [5]:

$$
\mathbf{Z}=\mathbf{Y C}_{\mathrm{WCM}}^{*} \text {, }
$$

where $\mathrm{C}_{\mathrm{WCM}}^{*}$ corresponds to the conjugate transpose matrix of $\mathbf{C}_{\mathrm{WCM}}$. From equations (2)-(4), the following statement results [2]:

$$
\mathbf{Z}=m g \mathbf{X I},
$$

where $\mathbf{I}$ is the identity matrix with rank $m$. From each element $z_{i}$ of vector $\mathbf{Z}$, the values assumed by the transmitted bits $x_{i}$ can be estimated by [4]

$$
x_{j+i-(m g-1)}=\operatorname{sgn}\left(z_{i}^{j}\right),
$$

where $\operatorname{sgn}(\cdot)$ is the signal function.

2.3. Modulation of Wavelet Coded Signals. Increasing the size of the wavelet coding matrix leads to an increase in the variance of symbols and their respective values, as shown in equations (8) and (9), as well as the diversity generated by the coding process [2]. Thus, the use of a conventional PSK modulation for the transmission of equally spaced wavelet symbols would result in a constellation characterized by an agglomeration of points over a unit radius circle.

Thus, an unusual PSK modulation is better recommended for many wavelet coding systems, where the distribution of the points over the circle is performed in order to optimize some desired metric, e.g., the system BER [7]. It is important to note that the use of a usual PSK constellation, with equispaced points in the unit radius circle, for the transmission of wavelet symbols would result in a loss of transmission system performance [2].

Obtaining BER-optimized constellations has been discussed in several works. For instance, a method for searching genetic algorithms for a truncated constellation suitable to wavelet coding is presented in [2]. The result is an unusual PSK modulation with $N_{p}$ PSK symbols. Table 1 presents the resulting constellation for wavelet encoding systems based on a $2 \times 128 \mathrm{WCM}$.

\section{Cyclostationary Processes}

A signal $x(t)$ is said to be second-order cyclostationary if there is a second-order nonlinear transformation linear $f(\cdot)$ capable of leading the resulting signal $y(t)=f(x(t))$ to contain sinusoidal components with infinite amplitude [14]. In practice, second-order cyclostationary signals have a periodic autocorrelation function in the following form [15]:

$$
R_{x}(t+T, \tau)=R_{x}(t, \tau),
$$

where $T$ is the period and $R_{x}(t, \tau)$ is the autocorrelation function defined by [16]

$$
R_{x}(t, \tau)=x\left(t+\frac{\tau}{2}\right) x^{*}\left(t+\frac{\tau}{2}\right),
$$

where $x^{*}(t)$ is the complex conjugate of $x(t)$, while the symbol $\bullet$ denotes the temporal mean operator defined by [16]

$$
x(t)=\lim _{T \longrightarrow \infty} \frac{1}{T} \int_{-T / 2}^{T / 2} x(t) \mathrm{d} t .
$$

When the theory of second-order cyclostationary processes is applied to spectral sensing, three important functions are used: the cyclic autocorrelation function (CAF), the spectral correlation density (SCD) function, and the alpha profile (AP) [9].

The autocorrelation function of a second-order cyclostationary process is periodic as it can be represented by a Fourier series. CAF can be defined as the coefficients resulting from the expansion of the autocorrelation function in terms of a Fourier series [14], i.e.,

$$
R_{x}^{\alpha}(\tau)=x\left(t+\frac{\tau}{2}\right) x^{*}\left(t+\frac{\tau}{2}\right) \exp (-j 2 \pi \alpha t),
$$

where $\alpha$ is the cyclic frequency, which is a discrete number. Therefore, a signal is second-order cyclostationary if the autocorrelation function is nonzero for any $\alpha \neq 0$ [16].

On the other hand, the SCD is defined as the Fourier transform of CAF, i.e., [16]

$$
S_{x}^{\alpha}(f)=\int_{-\infty}^{\infty} R_{x}^{\alpha}(\tau) \exp (-j 2 \pi f \tau) \mathrm{d} \tau,
$$

where $f$ is the signal frequency measured in $\mathrm{Hz}$.

The evaluation of SCD as expressed by equation (17) generates a surface over plane $(f, \alpha)$, which is symmetric with respect to both $f$ and $\alpha$. Considering the symmetries associated with the function, the projection of the SCD on an 
TABLE 1: Constellation for a wavelet encoding using a $2 \times 128 \mathrm{WCM}$ [2].

\begin{tabular}{lccc}
\hline Truncated set & Corresponding symbol & Angle & Probability of occurrence \\
\hline$\{0\}$ & 0 & $0^{\circ}$ & 0.0703 \\
$\{2,4,6\}$ & 4 & $22^{\circ}$ & 0.1967 \\
$\{-2,-4,-6\}$ & -4 & $-22^{\circ}$ & 0.1967 \\
$\{8,10,12\}$ & 10 & $60^{\circ}$ & 0.1430 \\
$\{-8,-10,-12\}$ & -10 & $-60^{\circ}$ & 0.1430 \\
$\{14,16,18\}$ & 16 & $90^{\circ}$ & 0.0789 \\
$\{-14,-16,-18\}$ & -16 & $-90^{\circ}$ & 0.0789 \\
$\{20,22,24\}$ & 22 & $110^{\circ}$ & 0.0330 \\
$\{-20,-22,-24\}$ & -22 & $-110^{\circ}$ & 0.0330 \\
$\{26, \ldots, 128\}$ & 28 & $125^{\circ}$ & 0.0134 \\
$\{-26, \ldots,-128\}$ & -28 & $-125^{\circ}$ & 0.0134 \\
\hline
\end{tabular}

orthogonal plane to $f$ is often calculated for $\alpha>0$, which is called AP [9],

$$
\rho(\alpha)=\frac{\max _{f}\left[S_{x}^{\alpha}(f)\right]}{\max _{f, \alpha}\left[S_{x}^{\alpha}(f)\right]} .
$$

3.1. Cyclostationary Sensing. The cyclostationarity sensing method is based on the fact that the Gaussian noise has no spectral correlation for a nonzero cyclic frequency [16]. From this principle, a simple method to check for communication signals in the spectrum range under analysis lies in detecting peaks in the alpha profile of the sensed channel $\alpha>0$ [9].

In this case, one possible way to obtain spectral sensing using cyclostationary process is to determine the value of a sensing metric $(\varepsilon)$ calculated as a function of the alpha profile according to [17],

$$
\varepsilon=\rho\left(\alpha=\alpha_{i}\right),
$$

where $\alpha_{i}$ is the value of the cyclic frequency defined as a function of the modulated signal format to be sensed by the architecture. Since the modulated signals have well-defined peaks in the alpha profile, the parameter $\alpha_{i}$ represents a cyclic frequency chosen to match the occurrence of such peaks in the alpha profile.

Making a decision in terms of the presence or absence of the communication signal occurs through a Bayesian test. Assuming that there is a modulated signal in the analyzed spectrum range, the sensing metric value will tend to be greater than zero $(\varepsilon>0)$. Otherwise, if there is no communication signal in the analyzed range, the sensing metric will tend to low values close to zero. Thus, assuming that the suboptimal threshold $(\delta)$ is known, which is calculated as a function of the desired false alarm probability, the proposed sensing architecture can perform the following test for the detection of communication signals in the analyzed spectrum:

(i) If $\varepsilon>\delta$, the communication signal will exist

(ii) Otherwise, the communication signal will be absent

3.2. Spectral Sensing of Signals Coded with Wavelet Matrices. Applying cyclostationarity metrics to signals containing a constellation whose patterns are similar as those described in Table 1 results in a shading of the cyclostationary

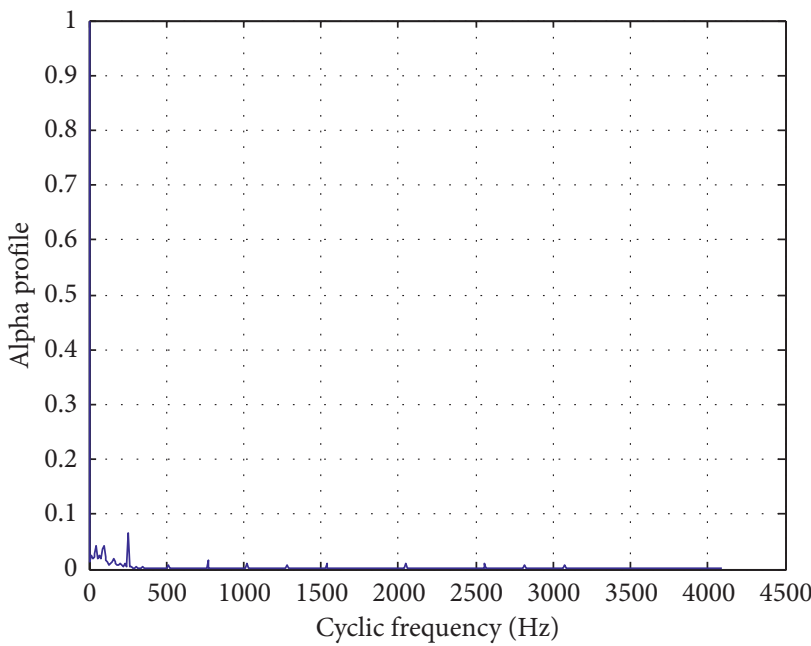

FIGURE 1: Alpha profile for wavelet symbols with the modulation scheme indicated in Table 1.

characteristics of the transmitted coded signal. Figure 1 represents the alpha profile calculated for a signal using the modulation described in Section 3.

On the other hand, empirical results indicate that, by adjusting the mapping of wavelet symbols in a different way, the spectral lines resulting from the analysis of cyclostationary characteristics of signals with wavelet coding are evidenced [17]. Therefore, wavelet coding can be used in a DSA scenario with spectral sensing by cyclostationary analysis. However, an adjustment should be made to the way wavelet symbols are modulated and transmitted over the wireless communication channel. However, such adjustment must be performed so that it does not impair the performance of the channel encoder, which is measured in terms of the BER.

The forthcoming section details a method for searching a wavelet constellation that provides optimized BER performance and spectral sensing capability based on genetic algorithms.

\section{Constellation Design for Wavelet Signal Sensing}

In this work, a novel modulation scheme for signals encoded with wavelet matrices is proposed to meet the following optimization criteria: 
(i) Peak amplitudes in the alpha profile

(ii) Low BERs

The search for such constellations was performed using genetic algorithms, given the versatility of this algorithm in solving predominantly stochastic problems [18]. The genetic algorithm was implemented in MATLAB $^{\circledR}$ environment using the toolbox described in [19].

No previous truncation of the symbols was considered in the design so that there is a point in the associated constellation for each generated wavelet symbol.

The characterization of the genetic algorithm is based on the following variables: (i) chromosome representation; (ii) generation of the initial population; (iii) fitness function; (iv) selection method and genetic operators; and (v) stopping criterion. Each one of the aforementioned aspects is described in the forthcoming subsections.

4.1. Chromosome Representation. In this work, each chromosome gene indicates the angle of the points in a PSK constellation as expressed in radians. Thus, the floating point representation becomes the most suitable approach for the adopted genetic algorithm. Each chromosome is represented by a vector $\mathbf{x}=\left[\begin{array}{llll}x_{1} & x_{2} & \cdots & x_{n}\end{array}\right] \in \mathfrak{R}^{n}$, where $x_{1}, x_{2}, \ldots, x_{n}$ are the angles of the designed PSK constellation. The constellations are always symmetrical with respect to the abscissa axis and located in the unit radius circle. These constraints are associated with the nature of the wavelet coding process and reduce the constellation search space.

4.2. Initial Population. The initial population was randomly created by selecting the genes of each chromosome from a uniform distribution $U(0, \pi)$.

4.3. Fitness Function. The fitness function is defined in terms of the system bit error probability and the amplitudes of the peaks that exist in the alpha profile of the wavelet-encoded signals. Since it consists in an optimization involving two optimality criteria, it is characterized as a multiobjective optimization problem.

Considering that it is desired to minimize the BER and maximize the peaks in the alpha profile of this constellation, this work proposes the use of a novel fitness function $F(\mathbf{x})$, which is composed of the linear combination of each one of the individual objective functions being defined as

$$
F(\mathbf{x})=w_{1} \rho\left(\alpha_{i}, \mathbf{x}\right)-w_{2} P_{e}(\mathbf{x}),
$$

where vector $\mathbf{x}$ represents the angles of the designed constellation, $\rho\left(\alpha_{i}, \mathbf{x}\right)$ is the value of the alpha profile of the constellation $\mathbf{x}$ calculated at the cyclic frequency $\alpha_{i}, P_{e}(\mathbf{x})$ is the bit error probability of the constellation $\mathbf{x}$, and $w_{1}$ and $w_{2}$ are the weights used in the multiobjective optimization process.

The alpha profile can be obtained using any cyclostationary analysis algorithm. In this work, this function was estimated based on the cyclic periodogram detection (CPD) algorithm described in [15].

The bit error probability of a wavelet-coding system can be determined from semianalytic functions. In this case, the constellation design was performed for Rayleigh channels with flat fading. Thus, the probability of bit error can be estimated from the analysis performed in [2].

It is important to note that wavelet coding provides diversity gain with unit coding rate and is therefore only interesting for fading channels. That is why we do not propose constellations optimized for AWGN channels.

4.4. Selection Method and Genetic Operators. The selection method and genetic operators aim at transforming the population over the generations, extending the search process until it reaches a stopping criterion. Table 2 indicates the chosen selection method and genetic operators.

4.5. Stopping Criterion. The stopping criterion determines when the genetic algorithm should stop its execution and resume the final population resulting from the optimization process. In this case, the stopping criterion corresponds to 900 generations.

\section{Simulation Results}

This section presents the simulation results for the analysis of wavelet coding in a DSA scenario. The constellation design considered a $2 \times 128 \mathrm{WCM}$ as the coding matrix. The modeled communication channel presents flat fading. In this case, the received signal vector $\mathbf{y}$ can be written as a function of the transmitted signal vector $\mathbf{x}$ as

$$
\mathbf{y}=\mathbf{h}^{\mathrm{T}} \mathbf{x}+\mathbf{n}
$$

where $\mathbf{h}$ corresponds to the attenuation vector of the received signal and $\mathbf{n}$ is the additive Gaussian white noise (AWGN) vector with zero mean and variance $N_{0} / 2$ per dimension, where $N_{0}$ is calculated from

$$
N_{0}=10^{-0,1\left(E_{b} / N_{0}\right)(\mathrm{dB})} \text {, }
$$

with $E_{b}$ being the bit energy of the transmitted signal.

5.1. Obtaining Optimized Constellations. For obtaining optimized wavelet constellations used in sensing, the fitness function used by the genetic algorithm as given by equation (20) is employed, where $w_{1}=25 \times 10^{-6}$ and $w_{2}=9.99975 \times 10^{-1}$ were adopted. Such parameters were determined empirically from various tests with different weights until adequate values were obtained for BER and amplitude of the alpha profile associated with the signals encoded by wavelets matrices.

According to [2], when calculating the bit error probability of wavelet-encoded signals, the modeling of a discrete channel is required. In this case, a channel model described by equation (21) was adopted. Assuming perfect interweaving of the wavelet symbols generated by the encoder, coefficients $\mathbf{h}$ are modeled as independent complex Gaussian 
TABle 2: Parameters of the selection operators of the genetic algorithm.

\begin{tabular}{lc}
\hline Operator & Description \\
\hline Selection & Normalized geometric selection. \\
Crossover & Heuristic crossover. \\
Mutation & Nonuniform multidimensional mutation. \\
\hline
\end{tabular}

random variables, whose real and imaginary parts have zero mean and variance equal to 0.5 . The simulations were performed based on the Monte Carlo method. The channel was simulated for a SNR of $17 \mathrm{~dB}$, and a sufficient number of signals was adopted to obtain the demodulation error matrix with good statistical accuracy. The received signals are demodulated using the maximum a posteriori estimation (MAP) rule.

To obtain the alpha profile for the calculation of the fitness function, a signal defined by the constellation to be optimized was simulated. The signal was modulated with the parameters described in Table 3. The alpha profile was estimated using the CPD algorithm with the parameters described in Table 4 . The parameters required by the genetic algorithm employed in the constellation design are listed in Table 5.

Table 6 presents the angles of the PSK constellation obtained with the genetic algorithm. Applying the cyclostationarity measurements to the constellation described in Table 6 leads to the alpha profile represented in Figure 2. It can be observed that the alpha profile has a spectral line located at $\alpha=f_{s} / 16$, with a higher amplitude than that of the alpha profile of the constellation proposed in [2] as shown in Figure 1.

To validate the performance of the communication system using the designed constellation, BER versus $E_{b} /$ $N_{0}(\mathrm{~dB})$ curves were estimated and plotted considering a Rayleigh channel model with flat fading. The simulations were performed based on the Monte Carlo method. For each point of the BER curves, a minimum of 200 bit errors was ensured in the estimation. Perfect interweaving of the wavelet symbols generated by the encoder is assumed, as well as that the receiver has perfect knowledge of the channel state. During reception, the signals are demodulated by the MAP rule.

The results obtained for the system BER are shown in Figure 3. As a comparison metric, the BER of the waveletencoded system using a constellation optimized in terms of the BER only is presented previously in the literature and described in [2] (the constellation is presented in Table 1). Besides, the BER of the system encoded with space-time block coding (STBC) is assumed to present the same spectral efficiency [20].

From the analysis of Figure 3, it is observed that the bit error probability curve for the constellation obtained in this work is higher than that in [2], which is an expected result, given that the constellation in this case was optimized for two purposes: minimizing the BER and increasing the peak amplitudes in the alpha profile. The results for the BER from the aforementioned constellation differ by approximately $2 \mathrm{~dB}$ from those obtained in [2], thus reinforcing the compromise between the primary user detection and system BER.
TABLE 3: Parameters of the simulated signal for the alpha profile estimated by the fitness function of the genetic algorithm.

\begin{tabular}{lc}
\hline Characteristic & Parameterization \\
\hline Carrier frequency & $f_{c}=1024 \mathrm{~Hz}$ \\
Sampling frequency & $f_{s}=4096 \mathrm{~Hz}$ \\
Number of baseband signal samples & $N=4096$ samples \\
Number of samples per symbol & $n_{\text {samp }}=16$ samples $/$ symbol \\
Roll-off factor & $B_{0}=0.5$ \\
\hline
\end{tabular}

TABle 4: Parameters of the CPD algorithm required for the estimation of the alpha profile by the fitness function of the genetic algorithm.

\begin{tabular}{lc}
\hline Characteristic & Parameterization \\
\hline Axis $\alpha$ resolution & $\Delta \alpha=8 \mathrm{~Hz}$ \\
Axis $f$ resolution & $\Delta f=16 \mathrm{~Hz}$ \\
\hline
\end{tabular}

TABLE 5: Parameters employed by the genetic algorithm.

\begin{tabular}{lc}
\hline Characteristic & Parameterization \\
\hline Crossover rate & $60 \%$ \\
Mutation rate & $8 \%$ \\
Population size & 150 individuals \\
Maximum number of generations & 900 \\
\hline
\end{tabular}

TABle 6: Optimized constellation for sensing purposes.

\begin{tabular}{|c|c|c|c|}
\hline Symbol & Angle & Symbol & Angle \\
\hline 0 & 0 & 66 & 0.92 \\
\hline 2 & 0.11 & 68 & 1.20 \\
\hline 4 & 0.29 & 70 & 1.75 \\
\hline 6 & 0.53 & 72 & 1.94 \\
\hline 8 & 2.79 & 74 & 2.99 \\
\hline 10 & 2.58 & 76 & 1.77 \\
\hline 12 & 2.32 & 78 & 1.24 \\
\hline 14 & 2.27 & 80 & 0.70 \\
\hline 16 & 2.19 & 82 & 2.41 \\
\hline 18 & 1.21 & 84 & 0.88 \\
\hline 20 & 1.35 & 86 & 0.78 \\
\hline 22 & 1.43 & 88 & 1.14 \\
\hline 24 & 1.88 & 90 & 0.95 \\
\hline 26 & 1.52 & 92 & 2.22 \\
\hline 28 & 1.82 & 94 & 0.22 \\
\hline 30 & 1.76 & 96 & 0.50 \\
\hline 32 & 1.69 & 98 & 1.45 \\
\hline 34 & 1.01 & 100 & 2.09 \\
\hline 36 & 1.55 & 102 & 0.75 \\
\hline 38 & 1.03 & 104 & 1.94 \\
\hline 40 & 1.50 & 106 & 2.56 \\
\hline 42 & 1.37 & 108 & 0.93 \\
\hline 44 & 0.66 & 110 & 1.53 \\
\hline 46 & 1.32 & 112 & 2.96 \\
\hline 48 & 1.17 & 114 & 0.86 \\
\hline 50 & 1.77 & 116 & 1.51 \\
\hline 52 & 1.75 & 118 & 1.23 \\
\hline 54 & 1.45 & 120 & 1.06 \\
\hline 56 & 1.46 & 122 & 1.65 \\
\hline 58 & 0.89 & 124 & 1.60 \\
\hline 60 & 2.36 & 126 & 2.54 \\
\hline 62 & 1.79 & 128 & 2.75 \\
\hline 64 & 2.97 & - & - \\
\hline
\end{tabular}




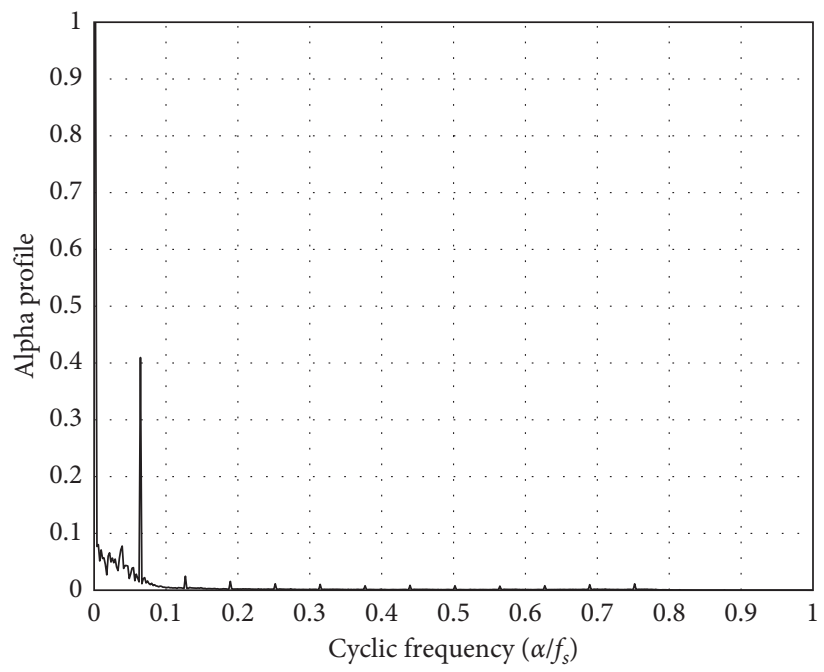

FIGURE 2: Alpha profile of the constellation used for sensing.

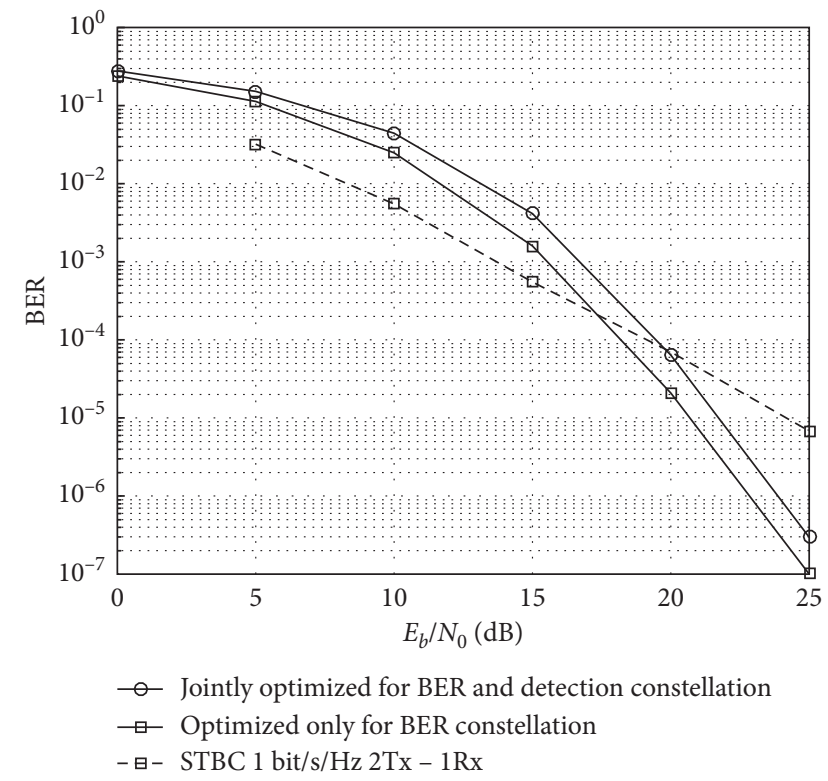

FIGURE 3: BER of the constellation used for sensing purposes compared with the optimized one in terms of BER and STBC.

Furthermore, looking at Figure 3, it is observed that since wavelet coding uses correlation decoding, the performance of this technique improves with high SNR, outperforming STBC coding. In addition, in STBC, diversity is achieved using multiple antennas, which yields better performance at lower SNRs.

5.2. Sensing Analysis of Wavelet-Coded Signals. Since the alpha profile of the designed constellation has a single peak at $\alpha=f_{s} / 16$, the sensing metric is represented by

$$
\varepsilon=\rho\left(\alpha=\frac{f_{s}}{16}\right) .
$$

The results of the cyclostationarity spectrum sensing were obtained considering the metric defined in equation (23). In all simulations, the signal was modulated with the parameters described in Table 3 . The parameters used in the CPD algorithm, which are necessary for the alpha profile calculation, are presented in Table 4. The communication channel was modeled as being Rayleigh correlated and generated using the Jakes method from 20 sinusoids so that the fading profile could be obtained [21]. The analyzed Doppler frequencies are $f_{d} T_{s}=0.0062$ and $f_{d} T_{s}=0.0031$.

Based on the international standard IEEE 802.22, which provides some requirements for DSA systems, a constant false alarm probability of $10 \%$ is desired [22]. This rate can be obtained by adjusting the comparison threshold used in the spectral sensing architecture. Since it was not possible to determine a mathematical relationship between the probability of false alarm and a given sensing threshold, a curve was employed instead in this work, as shown in Figure 4, which represents the relationship between such variables. This curve was obtained by simulating the probability of false alarm for the proposed architecture considering a white noise signal with unit variance and zero mean, where various threshold values were employed in a predetermined range $[0,0.30]$. For each threshold value, 300 simulations were carried out and the probability of false alarm was calculated for each case.

From Figure 4, a comparison threshold of $\delta=0.15$ was established, which leads to a false alarm probability of approximately $10 \%$. Therefore, the performance of the sensing architecture was evaluated considering this threshold.

Figure 5 presents the performance of the sensing architecture for the designed constellation. Each point of the curve was obtained through 3,500 simulations, which were divided into 35 groups of 100 tests. The detection probability was determined for each group, while the mean value of the detection probability was determined for the whole set considering a confidence interval of $95 \%$. 


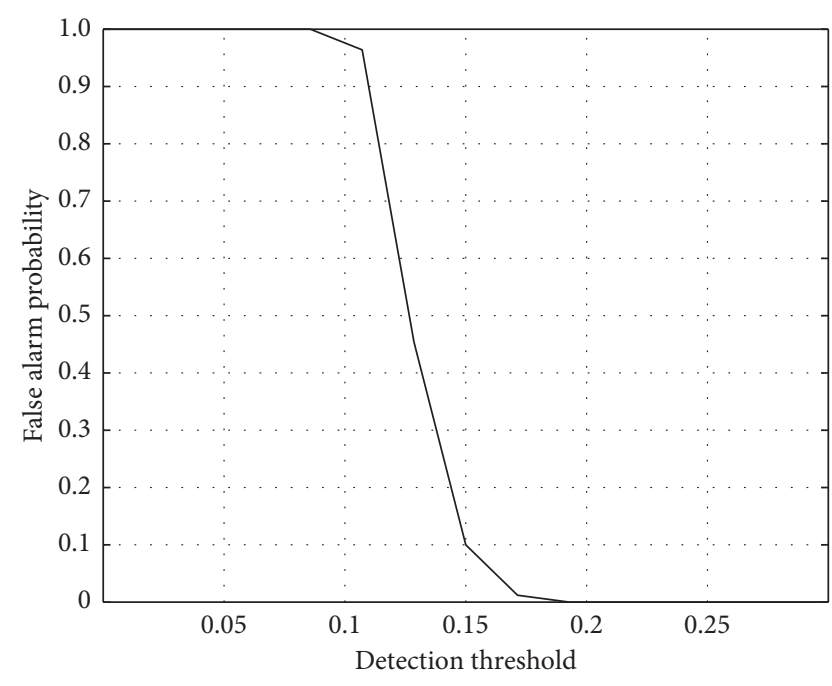

FIgURE 4: False alarm probability as a function of the detection threshold for the proposed architecture.

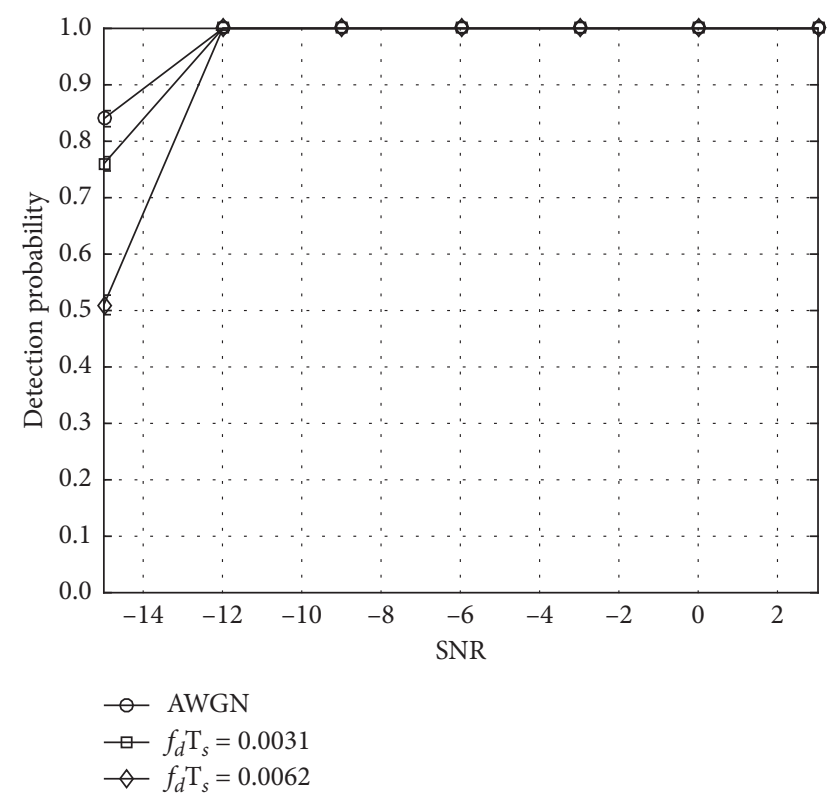

FIGURE 5: False alarm probability of the sensing method through cyclostationary analysis for a confidence interval of $95 \%$.

From the results shown in Figure 5, it can be stated that sensing the wavelet-coded signals leads to satisfactory results for channels with AWGN and low SNRs, i.e., in the order of $-12 \mathrm{~dB}$. Furthermore, it is observed that the detection probability of the constellation in AWGN channels is higher than that found in fading channels for the same SNR as expected, being in accordance with the results found in the literature [17].

The receiver operating characteristic (ROC) curves of the spectral sensing method are shown in Figures 6 and 7 for AWGN and Rayleigh channels with $f_{d} T_{s}=0.0062$, respectively. From such results, and considering the IEEE 802.22 standard, it is observed that the proposed constellation is efficiently sensed up to $-14 \mathrm{~dB}$ in AWGN channels and

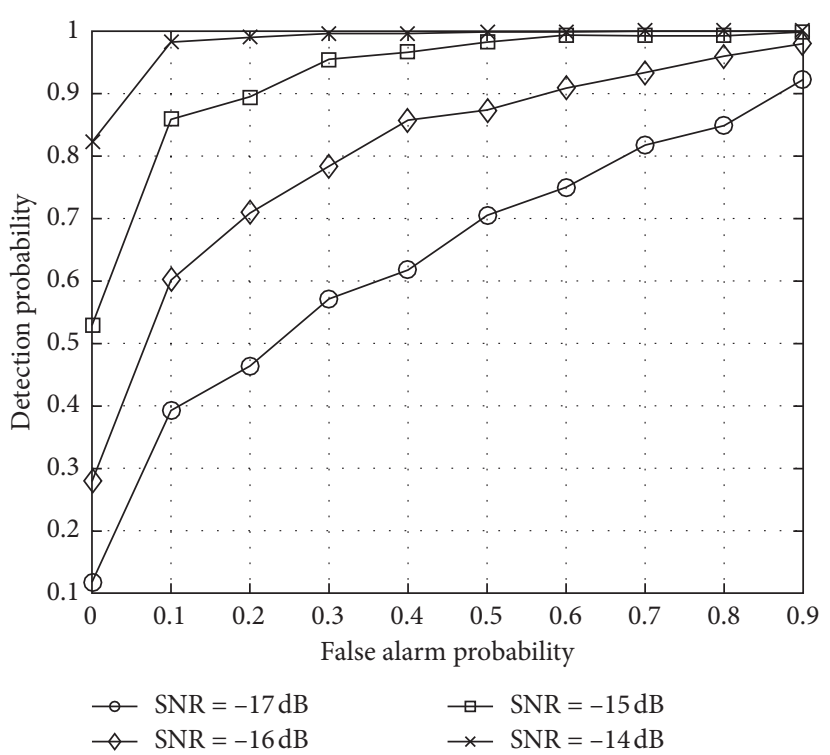

Figure 6: Receiver characteristic curve for AWGN channels.

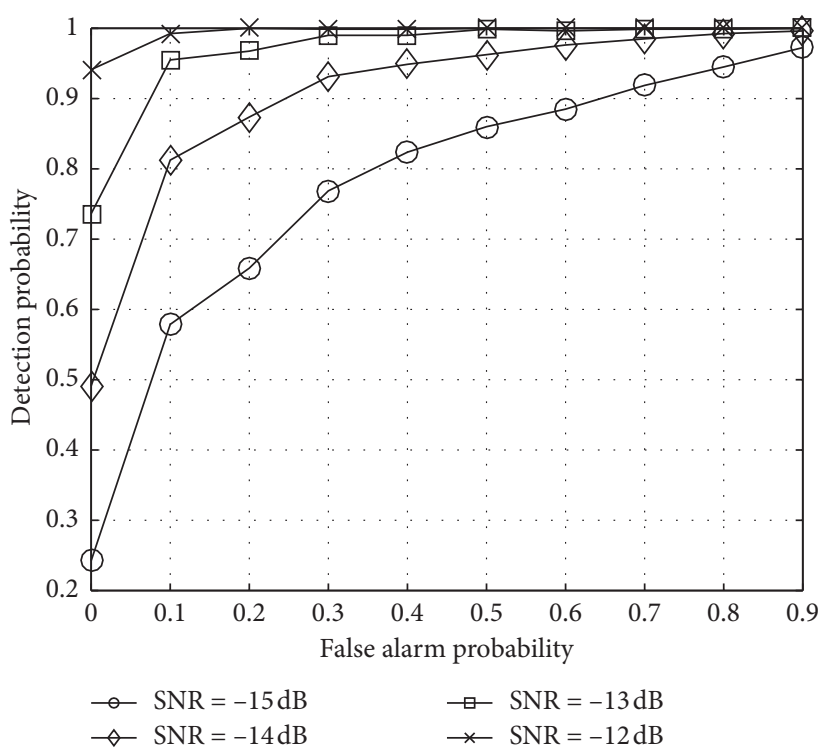

Figure 7: Receiver characteristic curve for Rayleigh channels.

$-12 \mathrm{~dB}$ in Rayleigh channels, thus demonstrating the robustness of this sensing method in environments with low SNRs.

\section{Conclusion}

This work presents a method for obtaining constellations suitable for sensing wavelet-encoded signals. From the simulation results, it can be stated that the proposed design method is quite efficient for defining the constellations while also providing good results in spectral sensing without compromising the system performance in terms of BER.

The main contributions of this work are as follows: (i) a study performed on the cyclostationary characteristics of signals encoded with wavelet matrices; (ii) presentation of a 
design method for novel modulation systems suitable for wavelet coding in spectral-sensing environments based on multiobjective optimization theory and genetic algorithms; and (iii) investigation of spectral-sensing techniques in systems using wavelet coding.

Future work aims at investigating the influence of some variables that were taken into account in the system modeling a priori, e.g., detection in environments with spectrum spreading, detection in channels characterized by frequency selectivity, and fading by shading.

\section{Data Availability}

The algorithms used and the constellations projected are available from the corresponding author upon request.

\section{Conflicts of Interest}

The authors declare that there are no conflicts of interest regarding the publication of this paper.

\section{Acknowledgments}

The authors acknowledge the valuable contributions of Dr. Edmar Candeia Gurjão (UFCG/Brazil), Dr. Adrião Duarte Doria Neto (UFRN/Brazil), and Dr. Vicente Angelo de Sousa Júnior (UFRN/Brazil). This study was financed in part by the Coordenação de perfeiçoamento de Pessoal de Nível Superior - Brasil (CAPES) - Finance Code 001, and technically supported by the High-Performance Computing Center at UFRN (NPAD/UFRN)

\section{References}

[1] T. S. Rappaport, Wireless Communications: Principles and Practice, Prentice Hall PTR, New Jersey, NJ, USA, 2006.

[2] L. F. Q. Silveira, Análise da Codificação Wavelet em Sistemas Sujeitos ao Desvanecimento Rayleigh Plano, Ph.D. thesis, Departamento de Engenharia Elétrica, UFCG, Campina Grande, Brazil, 2006.

[3] J. Proakis, Digital Communications, McGraw-Hill, New York, NY, USA, 1989.

[4] L. C. Cavalcante, Avaliação de Desempenho da Codificação Wavelet em Canais Seletivos em Frequência, Ph.D. thesis, Universidade Federal do Rio Grande do Norte, Natal, Brazil, 2017.

[5] M. A. Tzannes and M. C. Tzannes, "Bit-by-bit channel coding using wavelets," in Proceedings of the IEEE Global Telecommunications Conference-GLOBECOM'92, pp. 684-688, Orlando, FL, USA, December 1992.

[6] L. F. Q. Silveira, F. M. de Assis, and E. L. Pinto, "Performance of wavelet coding in systems with diversity over at and fast Rayleigh fading channels," in Proceedings of the XIX Brazilian Symposium of Telecommunications-SBrT, Fortaleza, Brazil, September 2001.

[7] L. F. Q. Silveria, F. M. Assis, and E. L. A. Pinto, "A new wavelet coded PSK system over time-varying flat fading," in Proceedings of the 2004 IEEE Workshop on Signal Processing Advances in Wireless Communications, Lisbon, Portugal, July 2004.

[8] L. F. Q. Silveira, L. G. Q. Silveira, F. M. Assis, and E. L. Pinto, "Analysis and optimization of wavelet-coded communication systems," IEEE Transactions on Wireless Communications, vol. 8, no. 2, pp. 563-567, 2009.

[9] T. R. Ferreira, "Modulação quantizada para sistemas com codificação wavelet sujeitos ao desvanecimento Rayleigh," Master thesis, Universidade Federal do Rio Grande do Norte, Natal, Brazil, 2009.

[10] L. C. P. Cavalcante, S. Rommel, R. Dinis, L. G. Q. S. Junior, L. F. Q. Silveira, and I. T. Monroy, "Performance evaluation of wavelet-coded OFDM on a $4.9 \mathrm{~Gb} / \mathrm{s} \mathrm{W}$-band radio-over-fiber link," Journal of Lightwave Technology, vol. 35, no. 14, pp. 2803-2809, 2017.

[11] F. Gama, L. Silveira, and A. Salazar, "Adaptive wavelet coding applied in a wireless control system," Sensors, vol. 17, no. 12, p. 2901, 2017.

[12] L. G. de Queiroz Silveira, "Wavelet-time diversity transmission in wireless systems: performance results," in Proceedings of the 2016 8th IEEE Latin-American Conference on Communications (LATINCOM), IEEE, Medellin, Colombia, November 2016.

[13] T. Yucek and H. Arslan, "A survey of spectrum sensing algorithms for cognitive radio applications," IEEE Communications Surveys \& Tutorials, vol. 11, no. 1, pp. 116-130, 2009.

[14] E. Costa, "Detectional and identification of cyclostationary signals," Master thesis, Naval Graduate School, Monterey, CA, USA, 1996.

[15] K. Kim, I. A. Akbar, K. K. Bae, J.-S. Um, C. M. Spooner, and J. H. Reed, "Cyclostationary approaches to signal detection and classification in cognitive radio," in Proceedings of the 2 nd IEEE International Symposium on New Frontiers in Dynamic Spectrum Access Networks, Dublin, Ireland, April 2007.

[16] W. Gardner, "Cyclostationarity in communications and signal processing," DTIC Document, IEEE Press, New York, NY, USA, 1994.

[17] P. T. V. Souza, "Análise da codificação wavelet em sistemas de rádio com acesso dinâmico ao espectro," Master thesis, Universidade Federal do Rio Grande do Norte, Natal, Brazil, 2017.

[18] L. Davis, Handbook of Genetic Algorithms, Van Nostrand Reinhold, New York, NY, USA, 1991.

[19] C. R. Houck, J. Joines, and M. G. Kay, A Genetic Algorithm for Function Optimization: A Matlab Implementation, NCSU-IE TR, The Mathworks, Natick, MA, USA, 1995.

[20] C. Tarokh, H. Jafarkhani, and A. R. Calderbank, "Space-time block coding for wireless communications: performance results," IEEE Journal on Selected Areas in Communications, vol. 17, no. 3, pp. 451-460, 1999.

[21] P. Dent, G. E. Bottomley, and T. Croft, "Jakes fading model revisited," Electronics Letters, vol. 29, no. 13, pp. 1162-1163, 1993.

[22] C. Cordeiro, K. Challapali, D. Birru, and S. Shankar, "IEEE 802.22: the first worldwide wireless standard based on cognitive radios," in Proceedings of the First IEEE International Symposium on New Frontiers in Dynamic Spectrum Access Networks, DySPAN 2005, pp. 328-337, IEEE, Baltimore, MD, USA, November 2005. 


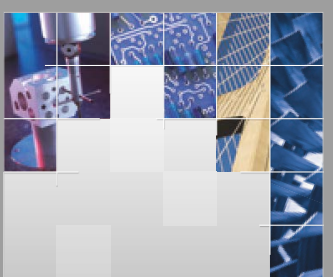

\section{Enfincering}
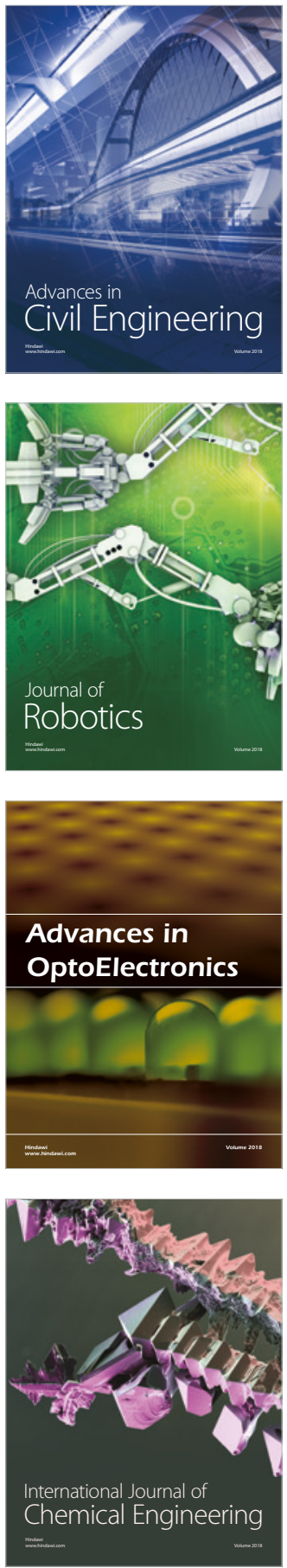

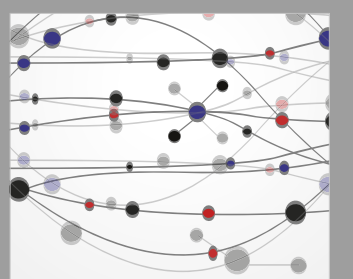

\section{Rotating \\ Machinery}

The Scientific World Journal

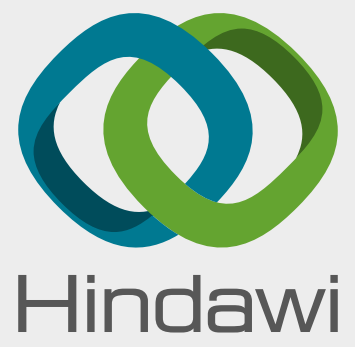

Submit your manuscripts at

www.hindawi.com
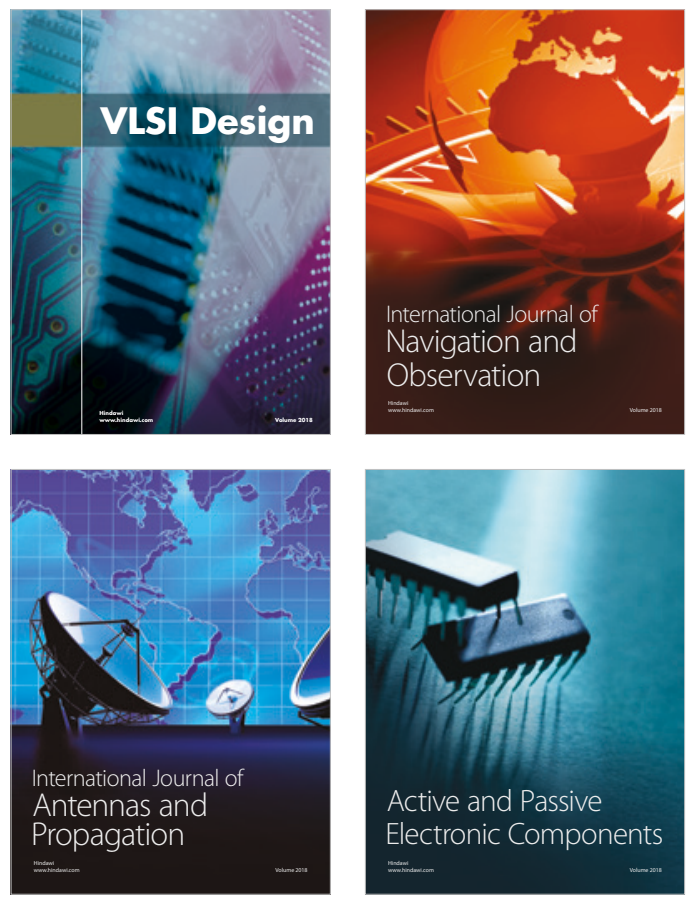
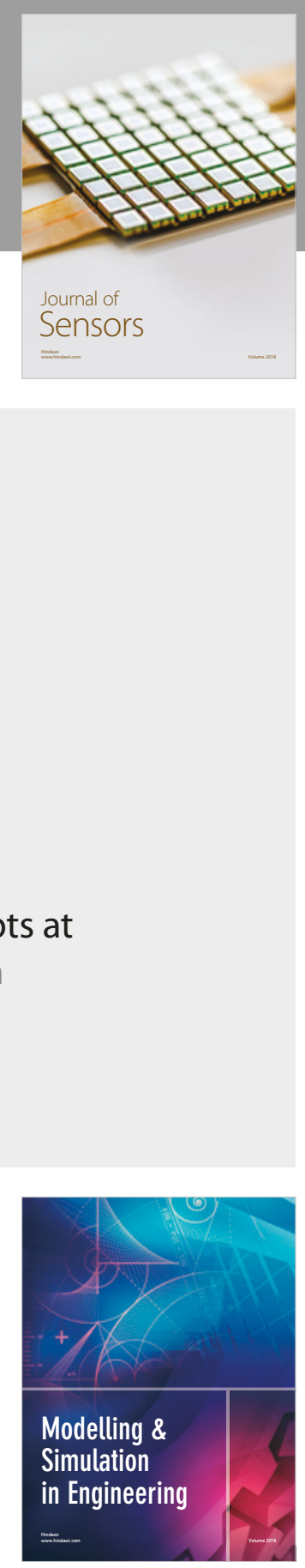

\section{Advances \\ Multimedia}
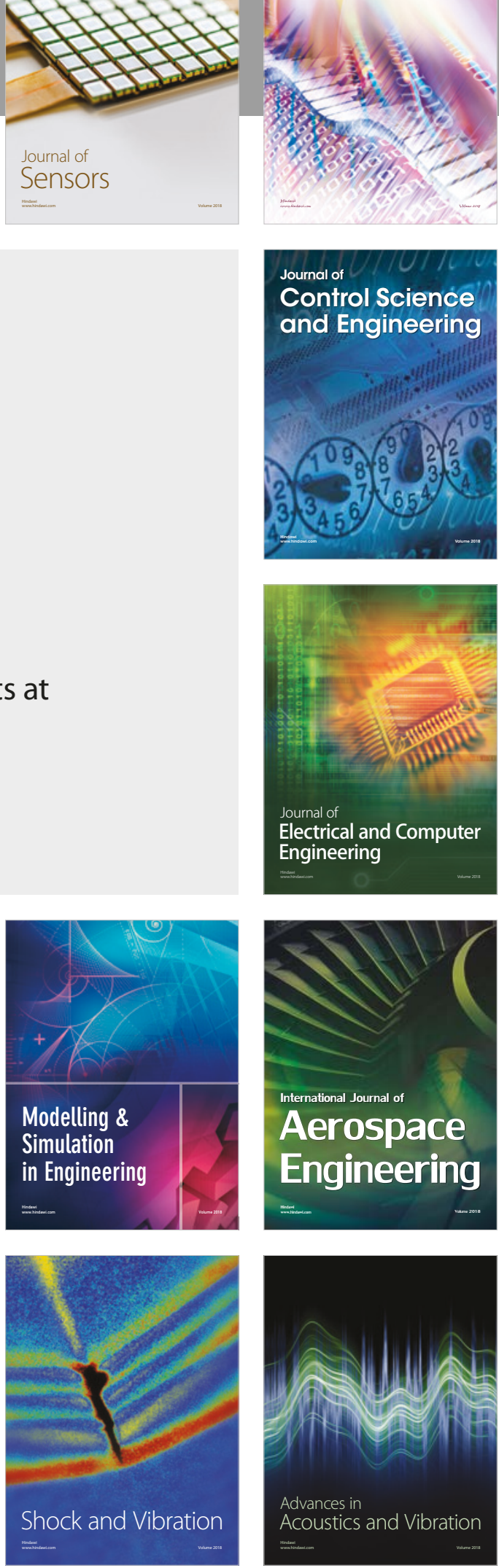